\title{
O estudo da morfologia urbana na Região Metropolitana de Campinas
}

The study of urban morphology in Campinas Metropolitan Region, Brazil

Evandro Ziggiatti Monteiro[a] [D, Silvia Aparecida Mikami Gonçalves Pina[a] ${ }^{[C}$,

Rodrigo Argenton Freire[a,b] [C], Daniel Teixeira Turczyn[a] (1)

[a] Universidade Estadual de Campinas (UNICAMP), Faculdade de Engenharia Civil Arquitetura e Urbanismo,

Campinas, SP, Brasil

[b] Centro Universitário Max Planck, Indaiatuba, SP, Brasil

Como citar: Monteiro, E. Z., Pina, S. A. M. G., Freire, R. A., \& Turczyn, D. T. (2020). 0 estudo da morfologia urbana na Região Metropolitana de Campinas. urbe. Revista Brasileira de Gestão Urbana, 12, e20190260. https://doi.org/10.1590/2175-3369.012.e20190260

\section{Resumo}

0 presente estudo tem como objetivo apresentar uma revisão atualizada das recentes pesquisas de morfologia urbana para um recorte específico do território brasileiro, a região metropolitana de Campinas (RMC). Como objetivo secundário, este trabalho também se propõe a identificar a influência da morfologia urbana como corrente internacional sobre os trabalhos da RMC, notadamente por meio das suas três principais escolas. 0 método consiste numa revisão da produção acadêmica realizada na RMC sobre morfologia urbana nos últimos dez anos, por meio da qual se buscam identificar os pesquisadores e grupos de pesquisa relacionados a essa ciência. Numa primeira fase, é utilizada a expressão de busca "Forma Urbana" E "Campinas" OU "Região Metropolitana de Campinas", nas bases de dados Google Scholar. Numa segunda fase, os currículos dos autores encontrados são escrutinados com um leque maior de palavras-chave. Na parte final do artigo, é exposto, como exemplo dessa produção da RMC, uma pesquisa com foco na morfologia urbana contemporânea. Os resultados apontam para certo descolamento de abordagens e métodos nos trabalhos da RMC em relação à corrente principal de morfologia urbana internacional, mas indicam, sobretudo, o crescimento desse diálogo, principalmente na esteira recente dos ISUFs e PNUMs.

Palavras-chave: Morfologia urbana. Região Metropolitana de Campinas. PNUM.

\section{Abstract}

This study aims to present an updated review of recent urban morphology research in a specific section of the Brazilian territory, the Campinas Metropolitan Region (RMC). As a secondary objective, this paper also aims to identify the influence of urban morphology as an international current upon the RMC research, notably through its three main schools. The method consists of a review of the literature produced in the RMC on urban morphology in the last 10 years through, which it was possible to identify

EZM é doutor em Engenharia Civil, e-mail: evanzigg@unicamp.br

SAMGP é doutora em Planejamento e Engenharia Urbana, e-mail: smikami@unicamp.br

RAF é doutor em Arquitetura, Tecnologia e Cidade, e-mail: rodrigo@odlab.cc

DTT é doutor em Arquitetura, Tecnologia e Cidade, e-mail: danieltturczyn@gmail.com 
researchers and research groups related to this science. In a first phase the search expression "Urban Form" AND "Campinas" OR "Metropolitan Region of Campinas" is used in the Google Scholar databases. In a second phase the curricula of the found authors are analyzed by a wider range of keywords. In the final part of the article, as an example of this RMC production, a research focusing on contemporary urban morphology is presented. The results point out to a certain detachment of approaches and methods in the work of the RMC in relation to the international urban morphology's mainstream, but the growth of this dialogue can be detected, especially following the most recent updates of ISUFs and PNUMs.

Keywords: Urban morphology. Campinas Metropolitan Region. PNUM.

\section{Introdução}

Costa (2007) e Costa \& Teixeira (2014) foram as autoras pioneiras a compor um panorama do desenvolvimento da morfologia urbana no Brasil, evidenciando que, desde a metade do século XX, já havia pesquisas em campos correlatos, agrupados no que Costa (2007) chamou "estudos urbanos". Entretanto, foi somente na primeira década do século XXI que os princípios e métodos das escolas de morfologia urbana começaram a ser incorporados de forma explícita no Brasil. A partir de 2007, ano de realização do International Seminar on Urban Form (ISUF)/Ouro Preto, houve aumento significativo no número de grupos de pesquisa e publicações nas diferentes regiões do país (Costa \& Teixeira, 2014). Antes disso e até 2015, data da primeira Portuguese-language Network of Urban Morphology (PNUM) no Brasil, as publicações encontravam-se esparsas nos diversos eventos científicos de planejamento urbano, notadamente no PLURIS ${ }^{1}$.

No entanto, observam-se ainda, nesses estudos, certa particularização e algum grau de isolacionismo. As dimensões continentais do país, somadas às diversidades socioculturais, econômicas e históricas de seus estados e regiões, são fatores importantes a serem considerados no desenvolvimento da morfologia urbana no Brasil, uma vez que impactam não apenas na pluralidade de cidades e paisagens, mas também na pluralidade de centros e grupos de pesquisa, procurando respostas mais adaptadas às especificidades locais. Embora o conhecimento e a aplicação dos métodos ligados às escolas tradicionais de morfologia urbana estejam atualmente presentes nos debates, isso ocorre de forma heterogênea no território nacional.

0 presente estudo tem como objetivo apresentar uma revisão atualizada das recentes pesquisas de morfologia urbana em um recorte específico do território brasileiro, a região metropolitana de Campinas (RMC). Como objetivo secundário, este trabalho também se propõe a identificar a influência da morfologia urbana como corrente internacional sobre os trabalhos da RMC, notadamente por meio das suas três principais escolas.

A RMC é uma das regiões metropolitanas mais novas do Brasil, surgindo no despontar da globalização técnico-científico-informacional iniciada na década de 1980 (Silva, 2008). Foi criada pela Lei complementar estadual no 870, de 19 de junho de 2000, e é constituída por vinte municípios: Americana, Arthur Nogueira, Cosmópolis, Engenheiro Coelho, Holambra, Hortolândia, Indaiatuba, Itatiba, Jaguariúna, Monte Mor, Morungaba, Nova Odessa, Paulínia, Pedreira, Santa Bárbara d'Oeste, Santo Antônio de Posse, Sumaré, Valinhos, Vinhedo e Campinas, sua sede. A área total ocupada pelos municípios é de $379.180,0$ hectares, com uma população estimada de 3.231 .033 pessoas (2019). Todas as cidades apresentam índice de desenvolvimento humano municipal (IDHM) alto ou muito alto, resultando em uma média de 0,792 para a RMC, sendo a terceira região metropolitana melhor ranqueada no Brasil.

Historicamente, o território da RMC desenvolveu-se vinculado ao transporte de riquezas e à infraestrutura correspondente, em contínuo processo de transformação. Assim, os vários ciclos

\footnotetext{
${ }^{1}$ Congresso luso-brasileiro para o Planeamento Urbano, Regional, Integrado e Sustentável.
} 
econômicos - o do ouro no século XVIII, o do café no século XIX, a industrialização nos anos 1950, a descentralização industrial na década de 1970 e a reestruturação produtiva nos anos 1990 - foram viabilizados pelas diferentes infraestruturas e evolução técnica que também se tornaram suporte espacial (Reis, 2006), permitindo novas escalas e dinâmicas urbanas que vêm produzindo importantes impactos regionais e alterações morfológicas sem precedentes.

Como particularidade, a RMC é uma das poucas regiões metropolitanas brasileiras que não está atrelada a uma capital de estado. Essa distinção de natureza político-territorial é capaz de influenciar simultaneamente a constituição morfológica dessas cidades, assim como as temáticas e preocupações dos pesquisadores e, portanto, a própria natureza das pesquisas. Trata-se de um território metropolitano com características interioranas, cujo macromodelo difere de regiões metropolitanas nucleadas, como a da maioria das capitais, para constituir uma região metropolitana na forma de constelação de cidades, na qual o protagonismo populacional e funcional do município-sede fica muito menos evidenciado (Baeninger, 2001).

Para compreender a disseminação da morfologia urbana nessa região, inicialmente se realizou uma revisão bibliográfica sistemática da literatura sobre os Estudos da Forma Urbana para identificar (i) os atuais grupos de pesquisa existentes no contexto da RMC; e (ii) as principais linhas metodológicas adotadas.

Numa etapa inicial, foi utilizada a expressão de busca "Forma Urbana" E "Campinas" OU "Região Metropolitana de Campinas" nas bases de dados Google Scholar, por meio do software Harzing's Publish or Perish. A busca foi limitada aos artigos, teses e dissertações produzidos nos últimos dez anos (de 2009 a 2019). A expressão foi aplicada aos campos: "título", "resumo" e "palavras-chave". Nessa primeira etapa, foram identificados 363 documentos - entre artigos, livros, teses e dissertações. Em um segundo momento, os documentos foram filtrados por todos os autores, primeiramente a partir dos (a) títulos e, em seguida, dos (b) resumos. A filtragem por títulos reduziu o número inicial para 193 itens e os resumos, para 98. Por fim, um estudo mais aprofundado dos documentos resultou em um total de 53 artigos, teses e dissertações. Foram removidos os resultados que apresentaram grande similaridade, ou seja, dois artigos com conteúdo similar desenvolvido pelo mesmo autor. Também foram retirados os resultados relacionados a livros e relatórios.

Essa primeira fase do levantamento revelou que o tema "forma urbana" tem uma produção vasta, diversificada e transversal a várias áreas do conhecimento. Entretanto, como também existe o interesse em identificar a influência das três escolas de morfologia urbana nesta produção acadêmica, numa segunda fase, a busca centrou-se num leque maior de palavras-chave diretamente aplicadas aos currículos dos pesquisadores mais recorrentes nos trabalhos encontrados da primeira etapa. Destacaram-se principalmente pesquisadores das duas maiores universidades da cidade de Campinas: Pontifícia Universidade Católica de Campinas (PUC-Campinas) e Universidade Estadual de Campinas (Unicamp).

Estudos morfológicos na cidade de Campinas foram desenvolvidos, de forma pioneira, por professores e pesquisadores da PUC-Campinas, a partir da década de 1980. Nas décadas seguintes, surgiram estudos da Unicamp ligados ao Instituto de Geociências e às três unidades que, em 1999, fundariam o primeiro curso de caráter público de Arquitetura e Urbanismo da RMC: Instituto de Filosofia e Ciências Humanas, o Instituto de Artes e a Faculdade de Engenharia Civil. Entretanto, toda a produção desse período pode ser classificada mais como estudos urbanos em que a questão da forma urbana é abordada do que estudos da forma urbana propriamente ditos.

Foi somente na primeira década dos anos 2000, com a parceria realizada entre a Universidade de São Paulo e a PUC-Campinas, nos estudos do QUAPÁ-SEL (Sistema de Espaços Livres) (Macedo et al., 2012) e com as primeiras participações dos pesquisadores da RMC nos ISUFs e PNUMs, é que a ciência ${ }^{2}$

\footnotetext{
2 Oliveira (2018, p. 1) define morfologia como a "[...] ciência que estuda a forma física das cidades, bem como os principais agentes e processos que as conformam ao longo do tempo. Devido à complexidade do seu 'objeto' de estudo, a morfologia urbana é de clara natureza interdisciplinar, recebendo contribuições de campos diversos como a arquitetura, o planejamento geográfico, a história, para citar alguns".
} 
da morfologia urbana e, simultaneamente, a disseminação das suas escolas começaram a despontar nos trabalhos acadêmicos da região. Ainda assim, nesse período, são raras as citações de autores importantes das três escolas principais, como M. R. G. Conzen, S. Muratori ou B. Hillier. As citações mais frequentes são de clássicos como Kevin Lynch, Gordon Cullen e Jane Jacobs. Muitas vezes, associadas ao termo "morfologia urbana", são encontradas citações de Lamas (2004), uma vez que o livro, apesar de ser mais um compêndio de desenho urbano, utiliza o termo no título. Assim, é importante destacar que, embora se tenha identificado na RMC a influência recente da corrente internacional de morfologia urbana, representada pela tradição do ISUF, a maioria dos trabalhos utilizou abordagens metodológicas híbridas ou mesmo independentes.

\section{Influência das escolas de morfologia urbana}

Na segunda fase do levantamento realizado para este artigo, foram escrutinados os currículos (plataforma Lattes) de 16 pesquisadores da RMC, cujos nomes haviam sido recorrentes na primeira fase. 0 escrutínio foi realizado utilizando palavras-chave relacionadas às três escolas principais de morfologia urbana, levando a uma amostragem de 46 trabalhos, entre produtos de mestrado/doutorado e publicações em eventos e periódicos. A Figura 1 traz, de forma diagramática, a amostragem dos trabalhos dos dois grupos principais, pesquisadores da PUC-Campinas e da Unicamp. Desses 46 trabalhos, 24 não utilizam citações diretas aos expoentes clássicos dessas escolas, embora demonstrem em seus procedimentos metodológicos e abordagens influência da morfologia urbana como ciência. Dentre os trabalhos que denotam influência direta das escolas, 14 foram identificados como influenciados pela morfologia inglesa, quatro, pela morfologia italiana e quatro, pela sintaxe espacial.

Um dos primeiros trabalhos da RMC a fazer referência a S. Muratori, considerado pioneiro da abordagem tipológico-projetual (escola italiana) de morfologia urbana, é o texto de Pereira (2012), docente da PUC-Campinas. Dentre os trabalhos orientados por essa pesquisadora, um dos mais recentes é a dissertação de Menegaldo (2019), que combina métodos da abordagem geográfica (inglesa) com a análise morfológico-tipológica (italiana) para desvendar tanto a tipologia de um palacete antigo quanto, com base na gleba remanescente desse imóvel, a evolução fundiária dessa parte da cidade de Campinas. 0 trabalho também adota métodos da História Social e da pesquisa documental para "[...] discutir o papel de um agente integrante da elite dominante do século XIX [...], reinterpretando fatos, abordagens e documentação primária" (Menegaldo, 2019, p. 17). Já Vercelli \& Tirello (2018), pesquisadoras da Unicamp, debruçando-se sobre o bairro do Bexiga na capital, logram, com base nas premissas teóricas da escola muratoriana, discutir as possibilidades de aperfeiçoamento dos inventários de bairros históricos.

Em relação à abordagem histórico-geográfica (escola inglesa), pelo levantamento realizado, a primeira referência a M. R. G. Conzen por pesquisadores da RMC seria o trabalho de Monteiro (2014). Sua apresentação, realizada no ISUF-2014 (Oliveira et al., 2014), na cidade do Porto, coincidiu com a apresentação de mais cinco trabalhos de pesquisadores da RMC: Silva \& Lima (2014), Piccinato \& Salgado (2014), Montezuma et al. (2014), Pezzuto \& Monteiro (2014) e Monteiro \& Turczyn (2014). Ao contrário do primeiro, esses cinco trabalhos apresentados no ISUF não citam referências relacionadas às escolas da morfologia urbana. 


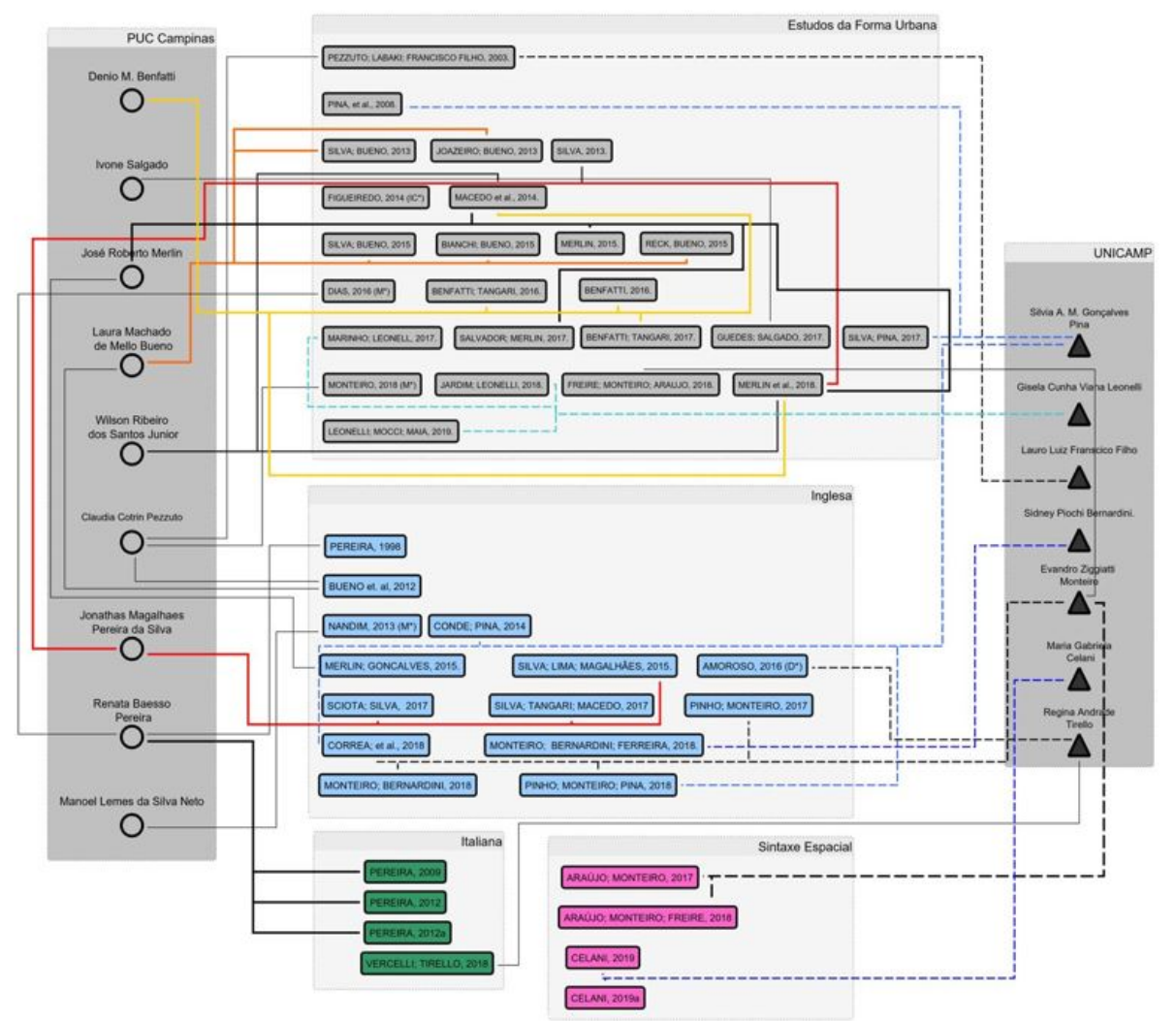

Figura 1 - Diagrama agrupando os pesquisadores da RMC por universidade de origem e correlacionando cada um aos seus respectivos trabalhos com ênfase em morfologia urbana. Fonte: Adaptado a partir de imagem gerada com auxílio do software Yed.

A influência da abordagem histórico-geográfica pôde ser observada em diversos trabalhos cujo escopo principal era o estudo do território, das formas urbanas, do ambiente urbano, da paisagem com vistas à sua aplicabilidade no planejamento, no desenho urbano e na gestão das cidades. Complementarmente, identifica-se um crescente interesse na adoção de tais perspectivas no âmbito do ensino de urbanismo e na discussão teórica. Exemplos são identificados nos trabalhos de Silva et al. (2016) e Monteiro (2017). Silva aponta a importância da morfologia urbana e da leitura de seus objetos como forma de compreender o "legado da ação humana sobre o território".

Em um mesmo sentido, Monteiro (2017, p. 29) destaca o uso possível da tipologia dos traçados para além da tradicional caracterização das regiões morfológicas e da evolução do tecido urbano, avançando para uma interpretação "[...] da própria história do poder na cidade, da disputa de classes sociais e grupos pela ordenação do seu território, pela construção de sua imagem simbólica". Desenvolvem-se também, pelos grupos de pesquisadores da RMC, estudos de inovação metodológica. Diante da raridade de bases cartográficas anteriores ao século XX na cidade de Campinas, Correa et al. (2018), da Unicamp, propõem também, segundo preceitos da escola inglesa, um método de conversão de registros escritos de cessão de terra em mapas rudimentares. 0 método promete permitir recompor a evolução das regiões morfológicas em cidades com pouca documentação gráfica. Ainda no ensino de urbanismo, destaca-se a proposta da gincana urbana, desenvolvida com os estudantes de arquitetura e urbanismo da Unicamp, que objetiva introduzir os estudantes aos clássicos da morfologia urbana, ao mesmo tempo que exercitam competências de percepção espacial na leitura de áreas centrais e núcleos históricos (Monteiro \& Bernardini, 2017; Monteiro et al., 2018).

Outras produções adotam a morfologia urbana e a leitura da paisagem com foco em aspectos metodológicos. Sciota (2016), Turczyn $(2013,2019)$ e Nandin (2013) interpretam a paisagem urbana contemporânea como reflexo dos recentes processos de urbanização e discutem o processo de espraiamento urbano, formação de loteamentos fechados e ocupação das margens das rodovias. Sciota 
(2016), por exemplo, analisa os núcleos urbanos fechados (NUFs) como caracterizadores da forma urbana e classifica-os com base em uma série de 12 categorias, entre as quais se destacam: (a) a relação com a área urbanizada; (b) o tamanho do NUF; (c) a configuração da testada; (d) a integração à paisagem urbana convencional e outras.

De forma geral, o trabalho de Sciota complementa a produção de Turczyn (2013) que investiga as mutações urbanas formadas ao longo dos eixos rodoviários que cortam o município de Campinas, em especial a Rodovia Dom Pedro I. Ao mesmo tempo, Nandin (2013), no Laboratório de Desenho de Estratégias Urbano-Regionais (PUC-Campinas), aponta tendências do desenvolvimento urbano recente do Eixo Noroeste da RMC - Campinas, Americana, Nova Odessa e Sumaré - ao longo da Rodovia Anhanguera. 0 que se verifica nos estudos que analisam esse território da RMC é o papel importante dos eixos viários que cortam/percorrem a RMC, em especial Campinas, na configuração de uma nova paisagem urbana altamente vinculada ao surgimento de conjuntos habitacionais e condomínios fechados (Pasquotto et al., 2018).

É importante salientar que a produção acadêmica dos pesquisadores da RMC é mais ampla quando o universo não se limita à RMC, como pode ser verificado nos anais da PNUM.

Por fim, um número reduzido de pesquisadores da RMC tem se dedicado à morfologia urbana a partir da sintaxe espacial. Pelo levantamento, foram encontrados apenas quatro trabalhos: Celani (2019a, 2019b), Araujo \& Monteiro (2017), Araujo et al. (2018).

\section{Outras abordagens independentes}

Uma parte dos trabalhos encontrados na primeira fase do levantamento, que se relacionavam com "Forma Urbana" E "Campinas" OU "Região Metropolitana de Campinas", foram considerados estudos da forma urbana de abordagem independente (24), uma vez que, além do fato de não trazerem citações aos clássicos de morfologia urbana, foi difícil detectar, em seus métodos, correspondências diretas com tais escolas. A ocorrência desses trabalhos não representa nenhum aspecto perturbador para a evolução da morfologia urbana como ciência na RMC nem no estado paulista. Ao contrário, demonstra que o tema tem despertado e impulsionado pesquisas e métodos os mais diversos e de forma complexa e rica. Nessa amostragem, também ocorrem produções mais diversificadas em termos de instituições (i.e., Universidade Paulista), provenientes de outras áreas, além de arquitetura e urbanismo.

Numa primeira classificação, foram identificadas pesquisas relacionadas a três linhas principais. A primeira delas corresponde à leitura histórico-morfológica dos processos de urbanização. Contempla os estudos que analisam o processo de urbanização por meio da narrativa histórica, identificando atores, eventos e mudanças de legislação. A segunda compreende os estudos que se concentram na análise crítica dos processos recentes de urbanização, com base na visão socioeconômica e na demografia. A terceira linha corresponde a uma série de trabalhos, notadamente conduzidos por pesquisadores da PUC-Campinas, que se alinham às importantes e contínuas pesquisas realizadas pelo laboratório Quapá, da capital de São Paulo, que pautam os estudos da forma urbana segundo o conceito de sistema de espaços livres (SEL).

Os estudos da forma urbana referentes ao processo histórico de urbanização da RMC e localidades próximas são oriundos, principalmente, dos grupos de Pesquisa "História das Cidades: Ocupação Territorial e Ideários Urbanos" estruturados pela PUC-Campinas e pelo grupo Terraplano, da Unicamp.

Dentre os estudos identificados, destacam-se o entendimento do processo de ocupação do território de Campinas entre os séculos XVIII e XX (Menegaldo, 2019; Krogh, 2013) e uma revisão dos processos de planejamento do final do século XX (Bernardini, 2018). Vale destacar o interesse dos mesmos grupos no estudo dos municípios localizados no interior paulista, não necessariamente pertencentes à RMC. Nesse sentido, é possível encontrar, dentro da mesma abordagem, estudos relacionados a municípios como Itu (Sampaio \& Salgado, 2016), Itapetininga (Guedes \& Salgado, 2017), Atibaia e Jundiaí (Salgado \& Pereira, 2014). Ainda explorando processos morfológicos 
históricos, Krogh (2013) investigou a reconfiguração urbana de Campinas em função da grande epidemia de febre amarela. A autora adotou a documentação primária como suporte para compreender essa reconfiguração. Por outro lado, Bernardini (2018) adota, também para Campinas, a sistematização de planos urbanos e outros dispositivos na análise da expansão urbana no período final do século XX.

Em relação à segunda linha, de estudos críticos dos processos contemporâneos de urbanização associados à forma urbana, diferentemente dos estudos voltados à paisagem urbana, estes adotam aspectos sociodemográficos na leitura do território e/ou a segregação socioespacial decorrente de políticas de habitação social. Nos estudos relacionados à segregação socioespacial, destacam-se as produções desenvolvidas pelo Núcleo de Estudos de População (Nepo-Unicamp). Os trabalhos de Cunha $(2011,2016)$ discutem a mobilidade espacial, mudanças na dinâmica territorial com base em dados demográficos, e.g. censos e levantamentos, e apontam também o surgimento de novas periferias, diversificadas e com uma população com maior renda.

Seguindo o mesmo contexto, Marins (2017) busca qualificar as áreas intermediárias entre o rural e o urbano com base em suas características socioeconômicas e demográficas. 0 autor aponta a importância de uma categoria intermediária entre o urbano e o rural no processo de compreensão do território da RMC e destaca, mais uma vez, a importância das rodovias como eixos estruturantes dos processos de urbanização e segregação socioespacial.

Bueno et al. (2012) discutem o impacto na forma urbana e o futuro das cidades com base nos processos mercadológicos associados ao território urbano. Ainda em relação a configuração demográfica e seus reflexos na forma urbana, Freire (2014) discute o conceito de cidades compactas e aplica indicadores relacionados à densidade populacional e à diversidade social para identificar localidades com características demográficas similares. De acordo com essa leitura, o autor busca identificar se localidades com mesmas características demográficas constituem também padrões morfológicos semelhantes.

Em relação à terceira linha, dos trabalhos alinhados aos estudos Quapá-SEL, destacamos Silva (2013), Merlin et al. (2018) e Benfatti (2016), que discutem o método de análise morfológica segundo o sistema de espaços livres e do território da RMC.

\section{Estudo das mutações urbanas}

O modelo atual e predominante do crescimento urbano das cidades da RMC é a periurbanização, por meio da implantação de tipologias habitacionais e comerciais fechadas que estruturam formas e paisagens urbanas bastante distintas daquelas encontradas nos tecidos urbanos tradicionais ou modernos que compõem as áreas urbanas mais antigas dessas cidades. A observação das características peculiares das dinâmicas de urbanização recente no contexto da RMC leva à seguinte questão: trata-se de um novo processo de cinturão periférico? Para responder a essa questão, foram utilizadas as categorias analíticas propostas por Solà-Morales (2002), uma vez que o processo aparentemente gera uma forma urbana muito mais fragmentada e desbalanceada do que os cinturões identificados pela teoria conzeniana (Pina \& Monteiro, 2010). 0 legado de Solà-Morales é descrito por Furtado (2009, p. 161) como um “[...] trabalho enigmático que possui capacidade genuína de criar uma cartografia que ilumina a cidade em sua complexidade contemporânea".

As cinco categorias propostas por ele, seguidas de seus sucintos conceitos, são: mutações - a forma das transformações; fluxos - a forma do movimento; habitações - a forma da residência; contenedores - a forma do intercâmbio; terras vagas - a forma da ausência. Segundo o autor, a categoria mutações é a primeira a ser apresentada porque é a mais adequada para entender as atuais dinâmicas de transformações súbitas das cidades. Nos estudos realizados e que serão aqui apresentados, o conceito de mutação urbana foi utilizado como chave para uma sintaxe de leitura espacial, com base na qual foi 
possível enfatizar e delimitar as aglomerações urbanas contemporâneas que compartilham do mesmo conjunto de características morfológicas, possibilitando a demarcação e o estudo dessas áreas.

Solà-Morales (2002) não explicita uma descrição clara de como seriam as formas das mutações urbanas, entretanto deixa implícito que as mutações representam os resultados formais da ocorrência conjunta das outras categorias. Cabe ressaltar, assim como fez o autor, que as categorias propostas não são um sistema fechado e completo de análise urbana, mas sim um sistema fértil e aberto para novas experimentações e aplicações.

Solà-Morales (2002) contextualiza o conceito de mutação urbana explicando que, desde o movimento renascentista até o organicista, o modelo orgânico-evolucionista havia sido utilizado para entender as relações de transformações urbanas, embasado nas teorias darwinianas, da evolução lenta e longa e do permanente reajuste entre morfologia e fisiologia. 0 autor exemplifica as mutações urbanas com os casos da reconstrução do centro de Beirute, da expansão urbana de Shangai, das transformações urbanas com a reunificação de Berlim, da renovação do centro de Bucareste, assim como do crescimento urbano da Cidade do México, de Brasília ou de Jedda. Para propor o conceito de mutação urbana, Solà-Morales (2002) também recorre à área da biologia e aponta que mutação é uma mudança aleatória no material genético de uma célula que produz alterações em uma ou mais características hereditárias, provocando uma ruptura com os mecanismos de herança, portanto uma mutação ocorre quando há alteração substancial que afeta tanto a morfologia como a fisiologia, não apenas de uma célula ou órgão, mas sim de todo o indivíduo.

0 autor não faz uma transposição do conceito da área da biologia para a área urbana. Fica claro que na biologia uma mutação ocorre quando há mudança no material genético de uma célula, produzindo alterações no tecido, no organismo e, consequentemente, no indivíduo. Pela perspectiva da morfologia urbana, pode-se inferir que a mutação é ocasionada pela mudança de um padrão na forma urbana que provoca transformações nos tecidos, bairros e em toda a cidade. Para Turczyn (2019) a alteração mais basal na forma urbana que caracteriza o desenvolvimento das mutações é a relação do espaço público com o privado, ou seja, a relação entre as tipologias residenciais e comerciais com as ruas, bairros e demais espaços de uso público. Essa alteração produz uma ruptura com a forma e a paisagem urbanas que antecederam as mutações, já que a relação entre as duas esferas é completamente diferente. Deste modo, Turczyn (2019) propõe uma paráfrase da definição da área da biologia, utilizada por Solà-Morales (1996), para a área urbana:

Mutação urbana significa uma transformação no modo de desenvolver territórios urbanos que produz alterações em uma ou mais características históricas da forma urbana, provocando uma ruptura com os tecidos urbanos preexistentes. Produzir uma mutação urbana é dizer que há uma alteração substancial que afeta a morfologia de uma aglomeração urbana - portanto, afeta as tipologias, as formas de implantação e as paisagens urbanas - e, consequentemente, de toda a cidade. (Turczyn, 2019, p. 201)

Turczyn (2019) aplicou o conceito de mutações urbanas para analisar a morfologia das áreas de expansão recente das cidades da RMC e localizou 25 aglomerações passíveis de serem classificadas como mutações, em sete cidades diferentes: 13 em Campinas, duas em Hortolândia, duas em Indaiatuba, duas em Paulínia, uma em Sumaré, duas em Valinhos e três em Vinhedo. Juntas, possuem 922 unidades de tipologias fechadas que ocupam uma área aproximada de 7.340 hectares. As tipologias habitacionais fechadas mais recorrentes são condomínios fechados (32\%), conjuntos habitacionais $(16 \%)$, loteamentos fechados $(13 \%)$ e vilas $(10 \%)$. As tipologias fechadas comerciais representam $13 \%$ do total de unidades encontradas. Cabe destacar a quantidade das tipologias comerciais: shopping-centers (11 un.), grandes lojas e hipermercados (50 un.), condomínios comerciais e industriais (33 un.) e malls (15 un.).

A análise macro da RMC evidenciou que a região que concentra a maior parte das mutações de Campinas está no entorno da rodovia Dom Pedro I, que faz parte do eixo que concentra a maior parte das mutações encontradas nas demais cidades. Esse eixo estende-se para noroeste em direção às cidades de Hortolândia, Paulínia e Sumaré, pelas rodovias Jornalista Francisco Aguirre Proença e 
Anhanguera, e para sudeste em direção às cidades de Valinhos e Vinhedo, pela rodovia José Roberto Magalhães Teixeira. As mutações situadas nesse eixo apresentam grandes possibilidades de expansão e conurbação, já que estão imersas em vazios urbanos, podendo, no futuro, concentrar uma vasta área conformada pelas características morfológicas das mutações urbanas (Figura 2).

A análise das mutações urbanas da RMC evidenciou que os elementos tradicionais que fundamentavam a urbanidade da cidade, como ruas comerciais, praças, lotes residenciais voltados para a rua, fachadas, marcos, quintais, portas, janelas, bancos, floreiras e, o mais simbólico, pedestres, cederam lugar a um conjunto tipológico e morfológico que enfatiza o espaço privado em detrimento do público, bem como o espaço do movimento rápido e irrestrito no lugar dos espaços de permanência, tornando os cidadãos reféns de um dentro sem fora. São aglomerações urbanas estruturadas por rodovias, avenidas arteriais, espaços gramados, grandes empreendimentos murados fora da escala do pedestre e do entorno, espaços de convívio e lazer privados e enclausurados, muros, grades, guaritas, aparatos de segurança, policiamento privado, outdoors, totens publicitários, grandes estacionamentos e apenas um equipamento público, o ponto de ônibus.

O espaço público resultante das mutações é mínimo e é estruturado pelos muros das tipologias fechadas. A divisão entre os espaços públicos e privados é reforçada por diferentes aparatos de segurança e pelo policiamento privado, já a permeabilidade urbana é reduzida e comprometida com a implantação fragmentada das tipologias fechadas e com a criação de ruas exclusivas para o acesso a elas. As calçadas apenas rodeiam os muros e não funcionam como meio de mobilidade. A natureza é negada e serve apenas como barreira ou limite para o desenvolvimento das mutações. A arquitetura é banalizada e os edifícios são repetidos sem qualquer cuidado com a composição da paisagem urbana ou com os bairros do entorno. 0 resultado final da soma dessas características é uma paisagem urbana homogênea, monótona, sem identidade, sem atrativos e pensada para ser um local de circulação, onde a permanência é rejeitada em todos os aspectos. 


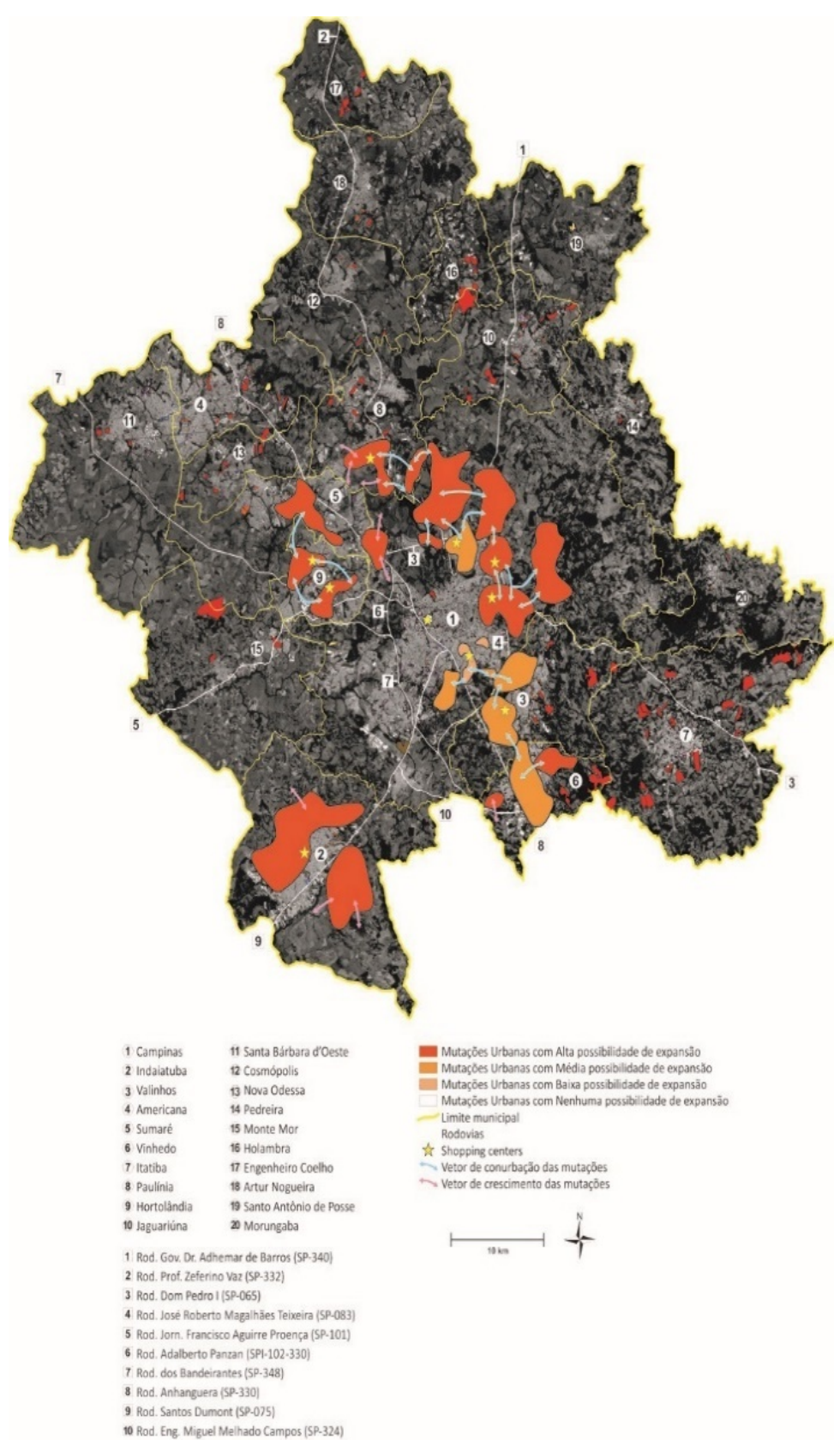

Figura 2 - Imagem da região metropolitana de Campinas (RMC), destacando-se as mutações urbanas e suas tendências de crescimento e conurbação. Fonte: Turczyn (2019).

A aplicação do conceito de mutações urbanas por Turczyn (2019) demonstrou uma abordagem morfológica plausível para estudar as áreas de expansão recente das cidades, no qual é possível analisar conjuntamente as dinâmicas de expansão urbana, o território de desenvolvimento, as tipologias residenciais e comerciais edificadas, os catalisadores, os vetores de crescimento, as formas que estruturam seu território e a paisagem urbana resultante. Trabalhar com as mutações urbanas permitiu identificar áreas de urbanização que compartilham características morfológicas muito próximas, possibilitando uma análise ampla e profunda. Portanto, analisar as mutações é analisar o último momento urbano das cidades, é olhar para áreas morfologicamente parecidas. Evidenciar essa forma de urbanização é chamar a atenção para um desenho do tecido urbano cada vez mais distante da produção de espaços públicos de qualidade, que sejam capazes de abrigar e instigar as inter-relações humanas, ou mesmo de espaços que conciliem a pauta de cidades mais sustentáveis, heterogêneas, inclusivas e voltadas às pessoas. 
O trabalho de Turczyn (2019) representa o estágio mais avançado dos estudos das mutações urbanas desenvolvidas pelo grupo de pesquisa em ensinagem, políticas públicas, sustentabilidade socioambiental e morfologia urbana do laboratório Fluxus da Unicamp. Porém, alguns estudos foram precursores a este: Turczyn \& Monteiro (2013a) e Turczyn (2013) evidenciaram as tipologias fechadas habitacionais e comerciais que conformam as mutações urbanas que estão nas margens da rodovia Dom Pedro I em Campinas; Turczyn \& Monteiro (2013b) apresentaram a possibilidade do desenvolvimento de mutações urbanas criativas em áreas de terras vagas de Campinas; Monteiro \& Turczyn (2018) fizeram um ensaio sobre a utilização das categorias analíticas de Solà-Morales para a análise urbana; Turczyn \& Monteiro (2018a) trouxeram o estudo das mutações urbanas na cidade de Campinas, evidenciando suas tipologias e padrões de formas de implantação; Turczyn \& Monteiro (2018b) apresentaram o estudo das mutações urbanas nas cidades que compõem a RMC, com exceção de Campinas, e seus padrões de paisagem.

\section{Conclusões}

Revisitando o que já concluíram Costa \& Teixeira (2014), ao analisar o quadro da morfologia urbana no Brasil, percebe-se que o contexto local da RMC não é tão diverso do identificado pelas autoras, no contexto nacional e naquele momento. Há, ainda, certo descolamento entre as abordagens, métodos e os "[...] conceitos de morfologia urbana que sustentam as pesquisas em influentes partes do continente europeu" (Costa \& Teixeira, 2014, p. 125). A princípio, o descolamento não deveria ser visto como fragilidade. Esse aspecto poderia significar independência das abordagens e dos métodos, ou, ainda, o pleno desenvolvimento de uma escola nacional ou regional de morfologia urbana. Entretanto, uma escola de conhecimento jamais se desenvolve alheia ao que se estuda nas outras. Pelo contrário: estabelece o diálogo, ou o debate, consonantes ou antagônicos. Este trabalho confirma que, no âmbito da RMC, um diálogo muito tímido se delineia com as demais escolas da morfologia urbana. Há pouco mais de uma década, nomes como M. R. G. Conzen, Saverio Muratori e Bill Hillier tinham sido citados em menos de meia dúzia de trabalhos da RMC. Por outro lado, pode-se afirmar que a influência do ISUF e, sobretudo, da PNUM, a rede lusófona de morfologia urbana, tem sido primordial para o estabelecimento do diálogo crescente, com uma participação cada vez maior de pesquisadores da RMC.

Nesse sentido, trabalhos como o de Turczyn (2019), de uma geração que já incorpora os benefícios do diálogo de ISUFs e PNUMs, revelam, ao mesmo tempo, as preocupações próprias do território da RMC enquanto metrópole interiorana que tem sofrido enormes pressões sobre sua estrutura morfológica. São cidades que havia pouco mais de um século guardavam tecidos urbanos compactos e dotados de edifícios produzidos sob influência dos colonizadores europeus. Eram cidades que contavam com infraestrutura férrea, sistemas de bondes e ruas caminháveis. As transformações que sofreram com a ascensão do rodoviarismo agora se somam às das mutações e de outras dinâmicas urbanas diferenciadas.

Neste quadro, é natural identificar como linha mestra das investigações de morfologia urbana no estado paulista, RMC incluso, uma certa obstinação pela apreensão da forma da metrópole contemporânea, pela busca incessante de como lidar com formas e processos que por sua escala espacial e temporal oprimem o habitante e sua vida na cidade. Entretanto, há nessa urgência, nessa pressa, um descuido a ser saneado. É preciso ainda ser minucioso com a compreensão dos períodos morfológicos desse território, com a correta valorização das tipologias arquitetônicas e com a simulação das possibilidades sintáxicas de malhas das cidades da região da metrópole campineira, entre tantos outros instrumentos, métodos e legados da ciência da morfologia urbana. 


\section{Referências}

Araujo, L. L. S., \& Monteiro, E. Z. (2017). Análise da vitalidade urbana em Poços de Caldas, MG: uma abordagem pela Sintaxe Espacial. In Anais da $6^{a}$ Conferência da Rede Lusófona de Morfologia Urbana (PNUM). Vitória: UFES.

Araujo, L. L. S., Monteiro, E. Z., \& Freire, R. A. (2018). Formas e usos de dois espaços públicos do centro de Poços de Caldas, MG: um resgate histórico a partir da sintaxe espacial. In PNUM 2018: A Produção do Território: Formas, Processos, Desígnios. Maringá: UEM.

Baeninger, R. (2001). Região Metropolitana de Campinas: expansão e consolidação do urbano paulista. In D. J. Hogan, J. M. P. Cunha, R. A. Baeninger, \& R. L. Carmo (Eds.), Migração e ambiente nas aglomerações urbanas (pp. 321-348). Campinas: MPC Artes Gráficas em Papel.

Benfatti, D. M. (2016). Campinas: sistemas de espaços livres e forma urbana. In Anais da VPNUM: Conferência da Rede Lusófona de Morfologia Urbana: Os Espaços da Morfologia Urbana. Guimarães, Portugal: PNUM.

Bernardini, S. P. (2018). 0 planejamento da expansão urbana na interface com a urbanização dispersa: uma análise sobre a Região Metropolitana de Campinas (1970-2006). urbe. Revista Brasileira de Gestão Urbana, 10(1), 172-185. http://dx.doi.org/10.1590/2175-3369.010.001.ao02.

Bueno, L. M. M., Tangari, V. R., Silva, J. M. P., Montezuma, C. M., \& Pezzutto, C. C. (2012). A morfologia resultante dos negócios de desenvolvimento urbano e o futuro das cidades. In Anais do VI Encontro Nacional da Associação Nacional de Pesquisa em Ambiente e Sociedade. Belém: UFPA.

Celani, M. G. C. (2019a). Cellular automata: towards possible applications in urban design education and practice| Autômatos celulares: visando possíveis aplicações no ensino e na prática do urbanismo. Oculum Ensaios, 16(2), 395-408. http://dx.doi.org/10.24220/2318-0919v16n2a4211.

Celani, M. G. C. (2019b). Towards urban densification. In International Conference on Computer-Aided Architectural Design Futures (pp. 489-503). Singapore: Springer.

Correa, R. H., Pina, S. A. M. G., Monteiro, E. Z., \& Ramme, J. (2018). Registros escritos no estudo da morfologia urbana: estudo de caso em Campinas, Brasil - 1815-1859. In Anais do PNUM 2018: A Produção do Território: Formas, Processos, Desígnios. Porto, Portugal: PNUM.

Costa, S. (2007). 0 estudo da forma urbana no Brasil. Arquitextos, 8, 087.05. Recuperado em 16 de dezembro de 2019, de https://www.vitruvius.com.br/revistas/read/arquitextos/08.087/220

Costa, S. A. P., \& Teixeira, M. C. V. (2014). The study of urban form in Brazil. Urban Morphology, 18(2), 119-127.

Cunha, J. M. P. D. (2011). Movilidad espacial, vulnerabilidad y segregación socioespacial: reflexiones a partir del estudio de la Región Metropolitana de Campinas. Notas de Poblacion, (93), 169-210.

Cunha, J. M. P. D. (2016). Aglomerações urbanas e mobilidade populacional: o caso da Região Metropolitana de Campinas. Revista Brasileira de Estudos de População, 33(1), 99-128. http://dx.doi.org/10.20947/S0102309820160006.

Freire, R. A. (2014). Densidade e diversidade: as dimensões de compacidade urbana (Dissertação de mestrado). Faculdade de Engenharia Civil, Arquitetura e Urbanismo, Universidade Estadual de Campinas, Campinas.

Furtado, G. (2009). Interpreting the contemporary metropolis: notes on the urban debate and on Ignasi Solà-Morales. Footprint, 3(2), 161-172.

Guedes, C. J., \& Salgado, I. (2017). Análise morfológica comparativa: a rede das vilas de Itapeva, Apiaí e Itapetininga fundadas no território paulista sob o governo do Morgado de Mateus. Labor e Engenho, 11(3), 296-312. http://dx.doi.org/10.20396/labore.v11i3.8648841.

Krogh, D. D. S. S. (2013). A reconfiguração urbana de Campinas no contexto das epidemias de Febre Amarela no final do século XIX (1880-1900) (Dissertação de mestrado). Pontifícia Universidade Católica de Campinas, Campinas.

Lamas, J. M. R. G. (2004). Morfologia urbana e desenho da cidade. Lisboa: Fundação Calouste Gulbenkian. 
Macedo, S. S., Queiroga, E. F., Galender, F. C., Campos, A. C. A., Custódio, V., Degreas, H., \& Gonçalves, F. M. (2012). Os sistemas de espaços livres na constituição da forma urbana contemporânea no brasil: produção e apropriação (QUAPÁSEL II). Paisagem e Ambiente, (30), 137-172. http://dx.doi.org/10.11606/issn.2359-5361.v0i30p137-172.

Marins, R. L. (2017). A expansão urbana da região metropolitana de Campinas através de categoria espacial intermediária entre rural e urbano (Dissertação de mestrado). Instituto de Filosofia e Ciências Humanas, Universidade Estadual de Campinas, Campinas.

Menegaldo, A. B. F. (2019). Entre o rural e o urbano: o Barão de Itapura como agente modelador da cidade de Campinas, SP (1869-1902) (Dissertação de mestrado). Pontificia Universidade Católica de Campinas, Campinas.

Merlin, J. R., Benfatti, D. M., Silva, J. M. P., \& Santos, W. R., Jr. (2018). Sistemas de espaços livres e morfologia urbana de Campinas. In S. S. Macedo, E. F. Queiroga, A. C. A. Campos, \& V. Custódio (Eds.), Quadro geral da forma urbana e do sistema de espaços livres das cidades brasileiras (1. ed., Vol. 1, p. 9-41). São Paulo: Editora FAU-USP.

Monteiro, E. Z. (2014). Urban form in diagrams. In ISUF Our Common Future in Urban Morphology: Book of Abstracts (p. 388). Porto: FEUP.

Monteiro, E. Z. (2017). A tipologia dos traçados urbanos como indicador de poderes concentrados ou dispersos. Revista de Morfologia Urbana, 5(1), 27.

Monteiro, E. Z., \& Bernardini, S. P. (2017). Gincana de urbanismo: um instrumento pedagógico de leitura da paisagem. In Anais da 6ª Conferência da Rede Lusófona de Morfologia Urbana (PNUM). Vitória: UFES.

Monteiro, E. Z., \& Turczyn, D. T. (2014). Five Elements Revisited: A Morphological Approach of Solà- Morales. In V. Oliveira, P. Pinho, L. M. Batista, T. Patatas (Eds.), ISUF Our Common Future in Urban Morphology: Book of Abstracts (p. 264). Porto: FEUP.

Monteiro, E. Z., \& Turczyn, D. T. (2018). The five categories of Solà-Morales as a legacy for reading the urban landscape. ACE: Architecture, City and Environment, 12(36), 73-90.

Monteiro, E. Z., Bernardini, S. P., \& Ferreira, C. L. (2018). Mapa e visiones seriales en centros históricos: un juego pedagógico de iniciación de morfologia urbana. In II Congreso Internacional ISUF-H: Ciudad y Formas Urbanas: Perspectivas Transversales (Vol. 8, pp. 103-112). Zaragoza: Universidad de Zaragoza, Instituición Fernando El Católico.

Montezuma, R., Pezzuto, C., \& Albuquerque, C. (2014). Dynamics interactions between urban form, green spaces and environmental quality in two metropolitan areas in Brazil. In ISUF Our Common Future in Urban Morphology: Book of Abstracts (p. 315). Porto: FEUP.

Nandin, C. L. C. (2013). Urbanização, morfologia e adensamento: o eixo noroeste da Região Metropolitana de Campinas (Dissertação de mestrado). Pontificia Universidade Católica de Campinas, Campinas.

Oliveira, V. (Ed.). (2018). Teaching urban morphology. Cham: Springer. http://dx.doi.org/10.1007/978-3-319-76126-8.

Oliveira, V., Pinho, P., Batista, L., \& Patatas, T. (Eds.). (2014). Our common future in urban morphology. Porto: FEUP.

Pasquotto, G., Silva, R., Lima, A., Argentone, L., Silva, M., Andrade, C., \& Sabalo, L. (2018). Análise morfológica das aglomerações residenciais horizontais intramuros na Região Metropolitana de Campinas. Revista de Morfologia Urbana, 6(1), 33-51.

Pereira, R. B. (2012). Tipologia arquitetônica e morfologia urbana: uma abordagem histórica de conceitos e métodos. São Paulo: Arquitextos.

Pezzuto, C., \& Monteiro, V. (2014). Assessment of the 'local climate zones' in area residential with low-rise buildings: the case of Campinas, Brazil. In ISUF Our Common Future in Urban Morphology: Book of Abstracts (p. 317). Porto: FEUP.

Piccinato, D., Jr., \& Salgado, I. (2014). Tenure of urban land: Structure, form and transformation of the original urban space of the city of Ribeirão Preto - SP, Brazil. In ISUF Our Common Future in Urban Morphology: Proceedings (pp. 788809). Porto: FEUP.

Pina, S. M. G., \& Monteiro, E. Z. (2010). A paisagem urbana como estratégia de governança de cidades sustentáveis. In $4^{\underline{0}}$ Congresso Luso-Brasileiro para o Planejamento Urbano, Regional, Integrado e Sustentável (PLURIS). Faro, Portugal: PLURIS. 
Reis, N. G. (2006). Notas sobre urbanização dispersa e novas formas de tecido urbano. São Paulo: Via das Artes.

Salgado, I., \& Pereira, R. B. (2014). A formação de núcleos urbanos no Brasil Colônia: procedimentos para elevar freguesias a vilas na Capitania de São Paulo na segunda metade do século XVIII. Cadernos de Pós-Graduação em Arquitetura e Urbanismo, 18(18), 1-21.

Sampaio, I. F., \& Salgado, I. (2016). Configuração urbana da vila de Itu no contexto da produção açucareira. Cadernos de Pós-Graduação em Arquitetura e Urbanismo, 15(2), 1-31.

Sciota, A. A. (2016). Critérios de avaliação de morfologia urbana em modelos de segregação residencial (Tese de doutorado). Pontificia Universidade Católica de Campinas, Campinas.

Silva, J. M. P. (2013). Os espaços livres de edificação como estruturadores da forma urbana. In Anais do PNUM 2013: Conference of the Portuguese Network of Urban Morphology (Vol. 1, pp. 535-552). Coimbra, Portugal: PNUM.

Silva, J. M. P., \& Lima, F. C. (2014). Urban form and land value: morphological types and patterns from spatial segregation in Campinas, SP, Brazil. In ISUF Our Common Future in Urban Morphology: Proceedings (p. 441). Porto: FEUP.

Silva, J. M. P., Benfatti, D., Moreira, T., \& Ribeiro, J. A. Z. M. T. (2016). Prática de ensino de planejamento urbano e regional: desenho como ferramenta de discussão e agentes como protagonistas. Arquitextos, 191(7), 1-17.

Silva, M. L. D. S., No. (2008). Campinas em dois tempos: fato metropolitano e desigualdades da metropolização globalitária em São Paulo. In M. A. D. Souza (Ed.), A metrópole e o futuro: refletindo sobre Campinas (Cap. 3, pp. 55-81). Campinas: Edições Territorial.

Solà-Morales, I. (1996). Presente y futuros. La arquitecturaenlasciudades. In XIX Congresso da União Internacional de Arquitetos (UIA). Barcelona: UIA.

Solà-Morales, I. (2002). Territorios (1. ed.). Barcelona: Editorial Gustavo Gili SA.

Turczyn, D. T. (2013). Mutação urbana em Campinas: sua forma e paisagem (Dissertação de mestrado). Faculdade de Engenharia Civil, Arquitetura e Urbanismo, Universidade Estadual de Campinas, Campinas.

Turczyn, D. T. (2019). Morfologia urbana contemporânea: contribuições para uma teoria das mutações urbanas (Tese do doutorado). Faculdade de Engenharia Civil, Arquitetura e Urbanismo, Universidade Estadual de Campinas, Campinas.

Turczyn, D. T., \& Monteiro, E. Z. (2013a). Mutações criativas: Campinas pode e precisa delas. In III Congresso Internacional de Cidades Criativas. Campinas: UNICAMP.

Turczyn, D. T., \& Monteiro, E. Z. (2013b). As tipologias das mutações urbanas nas margens da Rodovia Dom Pedrio I, em Campinas. In Anais do XV Encontro da Associação Nacional de Pós-graduação e Pesquisa em Planejamento Urbano e Regional (ENANPUR) (Vol. 15, No. 1). São Paulo: ANPUR.

Turczyn, D. T., \& Monteiro, E. Z. (2018a). Mutações urbanas em Campinas: suas tipologias e padrões de implantação. In PNUM 2018: A Produção do Território: Formas, Processos, Desígnios. Porto: PNUM.

Turczyn, D. T., \& Monteiro, E. Z. (2018b). Mutações urbanas na Região Metropolitana de Campinas: seus padrões de paisagem. In PNUM 2018: A Produção do Território: Formas, Processos, Desígnios. Porto: PNUM.

Vercelli, G., \& Tirello, R. A. (2018). As contribuições teóricas de Saverio Muratori para pensar o (re) inventário do histórico bairro da Bela Vista, São Paulo. In Anais do VENANPARQ (Vol. 3, pp. 5423-5439). Salvador: Editora Universidade Federal da Bahia.

Editores convidados: Vitor Oliveira (Universidade do Porto, Portugal) e Bruno Zaitter (PUCPR, Brasil)

Recebido: Dez. 16, 2019

Aprovado: Jan. 19, 2020 